\title{
Testing process for the WEAVE prime focus corrector lenses for the William Herschel Telescope
}

Dijana Bogunovic, Graeme Jonas, Dave Cochrane, Andrew Rakich, Peter Connor, et al.

Dijana Bogunovic, Graeme Jonas, Dave Cochrane, Andrew Rakich, Peter Connor, Abe Romeril, Jimi Romeril, Luke Gers, Eleanor Howick, Chris Young, Tony Reed, lan Tosh, Émilie Lhomé, Kevin M. Dee, Gavin Dalton , Scott Trager, Jose Alfonso Lopez Aguerri, Piercarlo Bonifacio, Antonella Vallenari, Esperanza Carrasco, Don Carlos Abrams, Kevin Middleton, "Testing process for the WEAVE prime focus corrector lenses for the William Herschel Telescope," Proc. SPIE 10700, Ground-based and Airborne Telescopes VII, 107003A (6 July 2018); doi: 10.1117/12.2312342

Event: SPIE Astronomical Telescopes + Instrumentation, 2018, Austin, Texas, United States 


\title{
Testing process for the WEAVE Prime Focus Corrector lenses for the William Herschel Telescope
}

\author{
Dijana Bogunovic ${ }^{\mathrm{a}}$, Graeme Jonas ${ }^{\mathrm{a}}$, Dave Cochrane $^{\mathrm{a}}$, Andrew Rakich ${ }^{\mathrm{b}}$, Peter Connor ${ }^{\mathrm{a}}$, Abe \\ Romerila $^{\mathrm{a}}$, Jimi Romeril ${ }^{\mathrm{a}}$, Luke Gers ${ }^{\mathrm{c}}$, Eleanor Howick ${ }^{\mathrm{d}}$, Chris Young ${ }^{\mathrm{d}}$, Tony Reed ${ }^{\mathrm{a}}$, Ian Tosh ${ }^{\mathrm{e}}$, \\ Emilie Lhoméf ${ }^{\mathrm{f}}$ Kevin M. Dee ${ }^{\mathrm{f}}$, Gavin Dalton ${ }^{\mathrm{e}, \mathrm{g}}$, Scott Trager ${ }^{\mathrm{h}}$, Jose Alfonso Lopez Aguerri ${ }^{\mathrm{i}}$, \\ Piercarlo Bonifacio ${ }^{\mathrm{j}}$, Antonella Vallenari ${ }^{\mathrm{k}}$, Esperanza Carrascol${ }^{1}$, Don Carlos Abrams ${ }^{\mathrm{f}}$, and \\ Kevin Middleton ${ }^{\mathrm{e}}$ \\ ${ }^{a}$ KiwiStar Optics/Callaghan Innovation, Lower Hutt 5010, New Zealand \\ ${ }^{\mathrm{b}}$ Mersenne Optical Consulting LTD, AZ, 85641, USA \\ ${ }^{\mathrm{c}}$ Formerly with KiwiStar Optics/Callaghan Innovation, Lower Hutt 5010, New Zealand \\ ${ }^{\mathrm{d}}$ MSL, Callaghan Innovation, Lower Hutt 5010, New Zealand \\ eRALSpace, STFC Rutherford Appleton Laboratory, OX11 0QX, UK \\ ${ }^{f}$ Isaac Newton Group of Telescopes, 38700 Santa Cruz de La Palma, Spain \\ 'Dept. of Physics, University of Oxford, Keble Road, Oxford, OX1 3RH, UK \\ ${ }^{\mathrm{h}}$ Kapteyn Institut, Rijksuniversiteit Groningen, Postbus 800, NL-9700 AV Groningen, \\ Netherlands

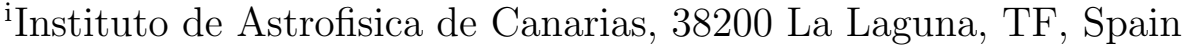 \\ ${ }^{j}$ GEPI, Observatoire de Paris, PSL Research University, CNRS, Place Jules Janssen, 92195 \\ Meudon, France \\ ${ }^{\mathrm{k}}$ Osservatorio Astronomico di Padova, INAF, Vicolo Osservatorio 5, 35122, Padova, Italy \\ ${ }^{1}$ INAOE, Luis Enrique Erro 1, Tonantzintla, Puebla, Mexico
}

\begin{abstract}
A new prime focus corrector for the WEAVE project for the William Herschel Telescope is being produced. The corrector consists of six lens elements, the largest being $1.1 \mathrm{~m}$ in diameter. It also incorporates an Atmospheric Dispersion Corrector. Testing procedures for the WEAVE prime focus corrector lens elements are described here. Critical issues encountered in practice, including the influence of the lens size, wedge and weight on the testing procedure are discussed. Due to large lens dimensions, a dedicated test tower and lens support system has been developed to measure the optical surface form errors of the concave surfaces and the transmitted wavefront of each lens. For some of the lens elements, sub-aperture measurements have been performed using an off-axis Hindle sphere and the resultant OPD maps have been stitched together. The challenge of testing a wedged lens with a combination of a long radius convex surface and a short radius concave surface has been resolved by using another lens from the system as an auxiliary lens. The practice of testing convex surfaces via internal reflection/transmission through the lens element has been avoided entirely in this case and some discussion justifying the choices of metrology approach taken is given. The fabrication and acceptance testing of the lens elements has been completed within the expected time and budget, and all elements have been shown to meet requirements.
\end{abstract}

Keywords: interferometric optical testing, prime focus wide-field corrector, large-size lens manufacturing and testing, atmospheric dispersion corrector

dijana.bogunovic@callaghaninnovation.govt.nz, Telephone: 6449313063 


\section{INTRODUCTION}

WEAVE (WHT Enhanced Area Velocity Explorer), a new multi-object survey spectrograph for the 4.2-m William Herschel Telescope (WHT) will be implemented later this year at the Roque de Los Muchachos observatory in the Canary Islands. It will enable spectroscopic follow-up observations of the fainter end of the Gaia astrometric catalogue and of the numerous sources from the medium deep LOFAR surveys. ${ }^{1,2}$ The instrument has a two-degree field-of-view prime focus corrector (PFC) with a 950-multiplex pick-and-place fibre positioner integrated into a top-end focusing assembly. It is feeding a medium and high resolution dual-band spectrograph, with full visible spectrum coverage for the first one and modest wavelength coverage for the latter. The detailed description of the testing procedure is presented here for six PFC lenses, manufactured at KiwiStar Optics, a business of Callaghan Innovation, New Zealand. The optical design and the manufacturing process for the PFC have been reported earlier. ${ }^{3,4}$ The WEAVE PFC consists of 6 lenses (Figure 1), where 4 of them make 2 doublets with incorporated wedge for the Atmospheric Dispersion Corrector.

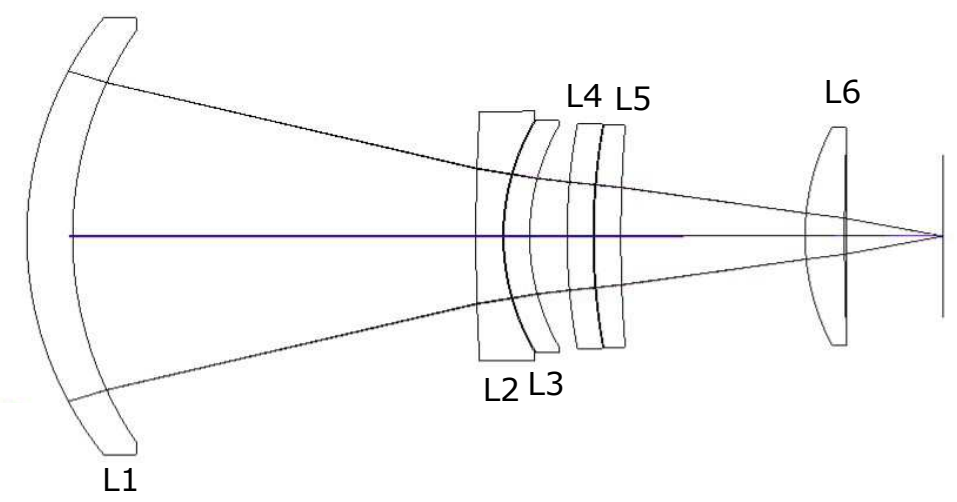

Figure 1. The PFC lenses. Materials: L1-Corning C79-80, L2-Ohara S-BSL7, L3-Ohara PBL1Y, L4-Ohara PBL1Y, L5-Schott N-BK7, L6-Ohara SK1300.

KiwiStar's lens manufacturing process combines traditional milling, grinding and pitch polishing processes, extended to accomodate lenses up to $1.5 \mathrm{~m}$ in diameter with high precision metrology. Blanks were milled using retrofitted manual milling machines, except for L1 which was pre-slumped and milled by the glass supplier.

\section{TESTING PROCESS}

The required optical testing residual errors had to meet the lens manufacturing specifications using the Standard Zernike coefficients in low (Z5-Z11), medium (Z12-Z190) and high (>Z190) spatial frequency ranges. The primary manufacturing and testing challange for the WEAVE PFC lenses was the size of the lenses, with L1 being $1.1 \mathrm{~m}$ in diameter and weighing $230 \mathrm{~kg}$. Wedged L2 has a large diameter and a long radius of curvature on its convex surface, which made it challenging to test. Wedged L3 and L4 have both surfaces tilted with respect to the PFC optical axis.

The initial WEAVE test setup had the lens optical axis in a vertical orientation with a fold mirror at $45^{\circ}$ and an ESDI-Mahr interferometer in horizontal orientation. ${ }^{4} \mathrm{~A}$ new, higher test tower has been developed in order to accomodate the interferometer in the vertical position, avoiding the use of the fold mirror. That simplified the alignment procedure and avoided any existing vignetting issues on the fold mirror. Each lens was placed in a purpose-built aluminium cell that allowed for $\mathrm{x} / \mathrm{y} / \mathrm{z}$ translation and tip/tilt of the lens. A continuous ring of $10-25 \mathrm{~mm}$ foam material is used at the tangent interface to protect the glass and create a compliant surface.

The practice of testing convex surfaces via internal reflection/transmission through the lens element has been avoided entirely and all lenses have been tested in double pass with Hindle Spheres as reflecting surfaces. This type of testing allowed extra degrees of freedom, as Hindle Spheres with different radii could be chosen to minimize residual spherical aberration, which would not be possible by testing the convex surfaces via internal reflection. 


\subsection{Lens 1 (L1)}

Due to its large diameter, 8 subaperture interferometric measurements have been performed for L1. A Hindle sphere with a clear aperture of $795 \mathrm{~mm}$ and a radius of curvature of $1250 \mathrm{~mm}$ was mounted on a rotary table at the base of a vertical test tower. The Hindle sphere is radially offset and tilted so it will later provide eight overlapping sub-aperture samples of the full aperture of L1 (Figure 2). The interferometer (ESDI H2000) and transmission sphere (MPF F/0.75) are mounted vertically in the test tower above the Hindle sphere. The Hindle sphere is then interferometrically tested at its center of curvature at eight 45-degree incremental rotational positions. This is achieved by rotating the Hindle sphere on the rotary table accordingly. These rotational positions are predetermined to provide eight overlapping sub-aperture samples of the full aperture of Lens 1 when it is included and tested later.

The cell/lens is then installed on a spider with supports separated by 120-degrees in the test tower between the interferometer and Hindle sphere at its appropriate location. The position of L1 with respect to the Hindle sphere and the interferometer was adjusted to produce minimum amount of induced alignment error for the first sub-aperture measurement. L1 was not adjusted again for the rest of the sub-aperture measurements for the entire stitched map.

The interferometer is used to test the optical combination (L1 and Hindle sphere) in double pass at the eight rotational positions (Figure 3). The Hindle sphere surface irregularity map is unique to each sub-aperture position and has been measured and then subtracted from each sub-aperture transmission measurement of Lens 1. IntelliWave OPD map transformations have been used to match the individual measurements of the Hindle sphere to the same digital mask diameters used in the transmission test with L1. This is due to slight image scale variation between testing only the Hindle sphere versus testing the Hindle sphere with L1 combination. Each sub-aperture measurement, for Lens 1 and the Hindle sphere, is an average of 8 interferometric measurements to reduce any errors generated by vibration and the local environment.
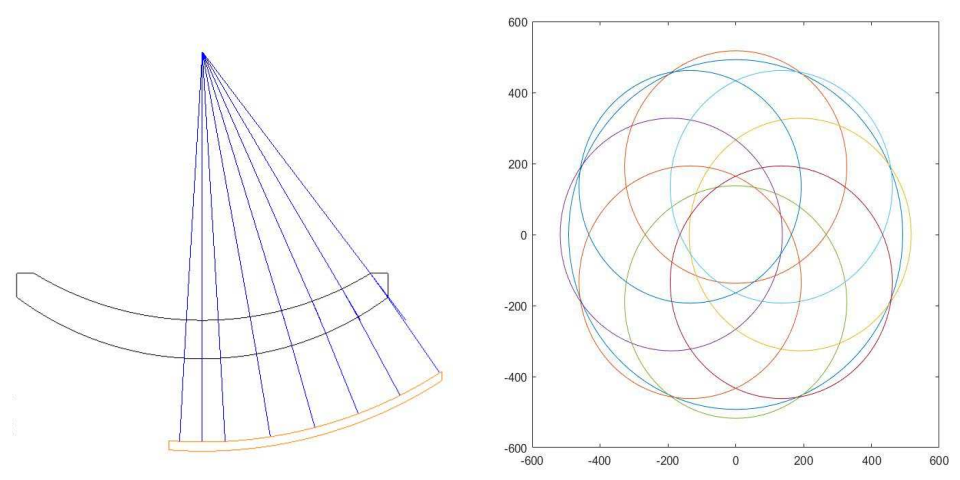

Figure 2. L1 test in double pass with $1250 \mathrm{~mm}$ radius Hindle sphere

The corrected sub-aperture measurements were then imported into ZBR Stitching Software from Arizona Optical Metrology (AOM). The software then tilts and pistons each sub-aperture map to generate a full aperture OPD map (Figure 4). ${ }^{5}$ The stitched map was then imported into Saguaro software for further analysis. ${ }^{6}$ Zernike coefficients were fitted to the OPD map and ZernikeTermRemoval Module was used to produce OPD maps at the different frequencies.

The concave surface of L1 has been measured in reflection. The weight of the lens has produced mount induced error, which was eliminated by the following method. Interferometric measures were taken of the lens at ten rotational clocking positions, with respect to the mounting cell $(0,36,72,108,144,180,216,252,288$, 324 degrees). 

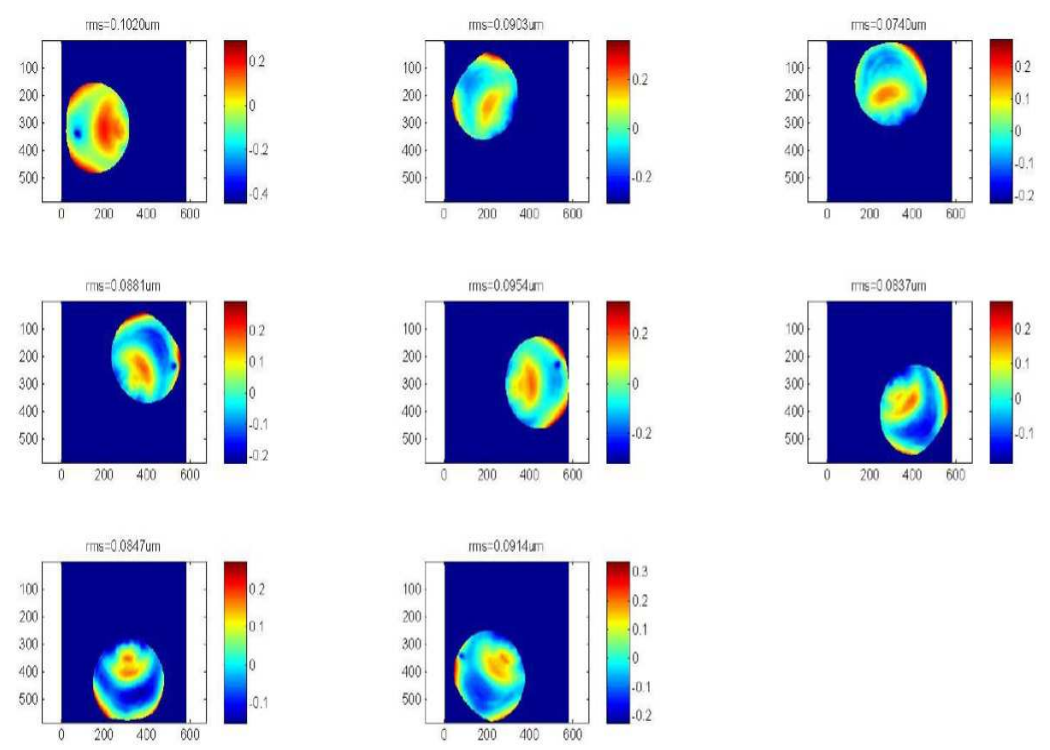

Figure 3. Sub-aperture OPD maps of L1. Tilt and focus have been subtracted.

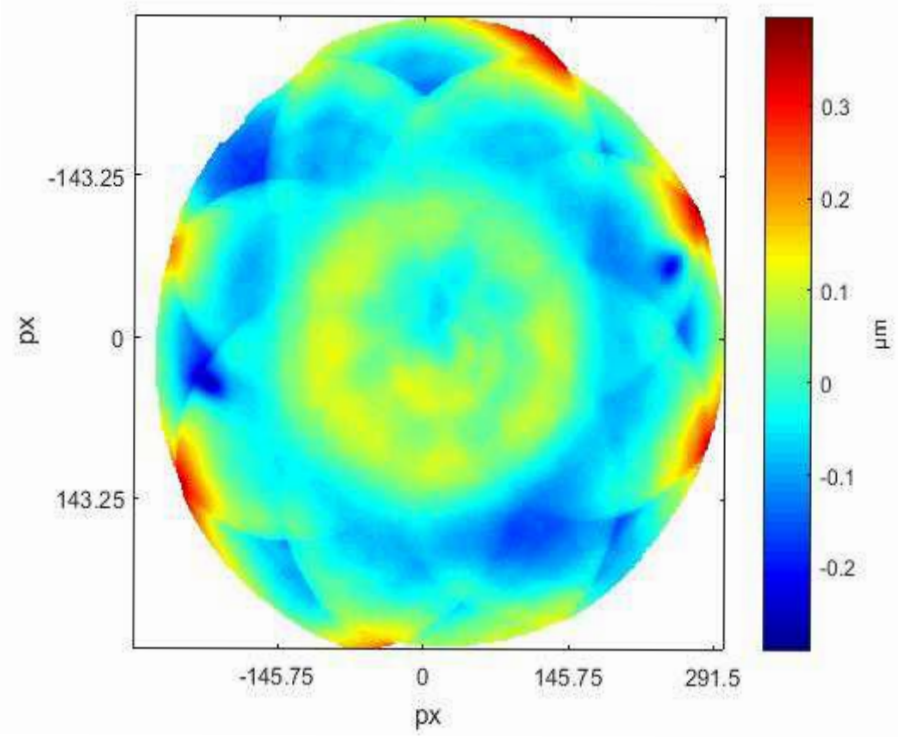

Figure 4. Total OPD map of L1. Tilt and focus have been subtracted. RMS=76.5 nm

Apart from the 0 degree clocking measure, all of the other measures were then digitally rotated back to the 0 degree origin, with the aid of fiducial markings and using Intelliwave software. The resulting back rotated measures, including the 0 degree origin measure, were then averaged using Intelliwave software. The resulting map is the surface profile of the lens with cell induced errors reduced to a level where it can safely be ignored.

\subsection{Lens 2 (L2)}

The concave surface of the L2 has been tested in reflection. The lens is tilted so that concave surface S2 is perpendicular to the interferometer's optical axis. No mount induced errors have been observed for this lens (138 $\mathrm{kg}$ ). Since both lenses L2 and L6 would have large residual spherical aberration when tested in transmission with 
KiwiStar's available Hindle spheres, further testing was performed on a combination of L2 and L6 in transmission using a computer-generated hologram (see subsection 2.6). Instead of distinguishing contributions from each of the two lenses, the final result met the tighter specification of the two, for the total transmitted wavefront error. It also met the tighter of the two specifications for low and high frequency range, while in the mid frequency range, it met the looser of the two specs. Additional testing of L2 convex surface was performed using a reference concave test plate and static fringe analysis, in order to determine the contribution of L2 in the mid frequency range.

\subsection{Lens 3 (L3)}

L3 was tested in reflection (concave surface) and transmission. The transmitted wavefront error is measured with a $1747.5 \mathrm{~mm}$ radius Hindle sphere (395 $\mathrm{mm}$ diameter) and a MPF F/0.75 reference transmission sphere. The OPD map is a result of six stitched transmitted OPD maps. The Hindle was measured by itself first on-axis and then at the off-axis sub-aperture positions. This allowed the optical axis (and the rotational axis) for the off-axis Hindle to be established, reducing alignment errors and establishing a reference plane.

The expected wavefront, as reported by Zemax, is then subtracted from the stitched wavefront data to generate the final transmitted wavefront error. Romer absolute arm (Hexagon Manufacturing Intelligence) was used to determine required distances and angles between the Hindle sphere and wedged lens.

\subsection{Lens $4(\mathrm{~L} 4)$}

The testing procedure for L4 is similar to the one performed for L3. The Hindle sphere was offset, decentered and tilted, to match the surface of the full size parent Hindle and reference measurements were taken for each sub-aperture position. Then the rotational axis of the Hindle was tilted an additional $0.45^{\circ}$ to counter the wedge of the L4 (Figure 5). The rotational inclined plane was created using a tilting rotary table. Z5 to Z8 (Fringe

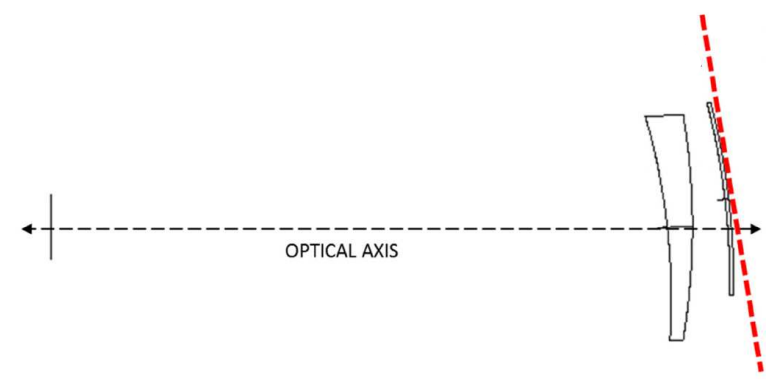

Figure 5. L4 wedge and additional Hindle sphere tilt angle. The angles are exaggarated.

Set) coefficients were used to fine tune the alignment of the lens to the Hindle and interferometer.

\subsection{Lens 5 (L5)}

Concave surface of L5 was ground to the best fit radius by KiwiStar Optics, and then aspherized and tested by Precision Asphere Inc. Available Hindle spheres were not sufficient to produce a null test, so a ComputerGenerated Hologram (CGH) was used for the double pass test.

\subsection{Lens 6 (L6)}

While the aspheric concave surface of L6 was tested (and manufactured) by Precision Asphere Inc, the testing in double pass was performed for L2 and L6 together using a CGH, a $1250 \mathrm{~mm}$ radius Hindle sphere and a MPF F/1.5 reference transmission sphere (Figure 6). The lenses are mounted in a single aluminium cell with a steel ring as a tangent interface. For the transmission test of Lenses 2 and 6 the Hindle sphere is tilted to -0.235 degrees to compensate for the wedge in Lens 2. The alignment of L2 and L6 was adjusted to minimize and match the Zemax reported wavefront error, using Zernike terms Z5, Z6, Z7, and Z8 (Fringe Set), for the 

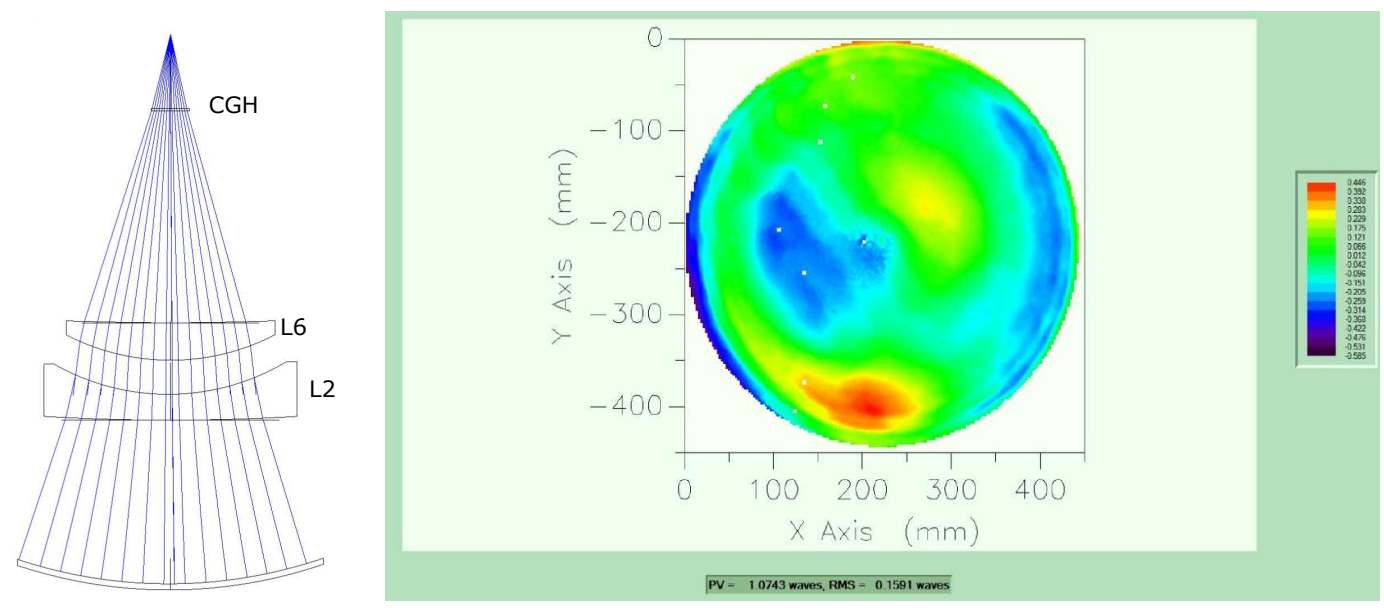

Figure 6. Test layout and OPD map for Lens 2 and 6 in double pass with $1250 \mathrm{~mm}$ radius Hindle sphere

entire test by adjusting the decenter ( $\mathrm{x}, \mathrm{y}$, and $\mathrm{z}$ ) of L2 and L6 with respect to the Hindle sphere (and hence the interferometer).

Mount induced error has not shown itself to be an issue.

\section{CONCLUSION}

The presented set of interferometric tests have been chosen in order to optimize testing time, complexity and budget without compromizing the precision of delivered results. All surfaces have shown to be within required tolerances in all frequency ranges.

\section{ACKNOWLEDGMENTS}

The authors would like to thank Callaghan Innovation Mechanical Workshop staff and NZ Measurements Standards Laboratory staff for their input on the development on the testing facility.

\section{REFERENCES}

[1] Dalton, G. B. et al., "WEAVE: the next generation wide-field spectroscopy facility for the William Herschel Telescope," Proc. SPIE, Volume 91478446 (2012).

[2] Dalton, G. et al., "Project overview and update on WEAVE: the next generation wide-field spectroscopy facility for the William Herschel Telescope," Proc. SPIE, Volume 9147 (2014).

[3] Agócs, T. et al., "Final optical design for the WEAVE two-degree Prime Focus Corrector," Proc. SPIE, Volume 9147 (2014).

[4] Lhomé, E. et al., "Manufacturing process for the WEAVE Prime Focus Corrector optics for the 4.2 William Hershel Telescope," Proc. SPIE, Volume 9912 (2016).

[5] Zhao, C. and Burge, J., "Stitching of off-axis sub-aperture null measurements of an aspheric surface," PROC. SPIE, Volume 7063 (2008).

[6] Kim, D., Lewis, B., and Burge, J., "Open-source data analysis and visualization software platform: SAGUARO," Proc. SPIE, Volume 8126 (2011). 